\title{
Computer-aided estimation of psychophysical thresholds by Wetherill tracking
}

\author{
THOMAS R. CORWIN \\ Center for Visual Science, University of Rochester, Rochester, New York 14627
}

ROBERT T. KINTZ

BRAG Microcomputers, Inc., Rochester, New York 14607

and

\author{
WILLIAM J. BEATY \\ University of Rochester, Rochester, New York 14627
}

\begin{abstract}
We describe a microcomputer implementation of an efficient procedure to obtain psychophysical thresholds at any of three response-probability levels, by Wetherill tracking. The procedure yields reliable 50\% thresholds (average coefficient of variation $=5.1 \%$ ) based on about 30 responses. Thresholds obtained in this way correlate highly with constant stimulus measurements requiring six times as many responses. Computer-assisted Wetherill tracking should find wide application in psychophysical screening tests.
\end{abstract}

In a large class of psychophysical experiments, subjects are presented with a series of stimuli that vary along a particular dimension, and they are required to respond to each stimulus level with one of two mutually exclusive responses. When the goal is to measure the subject's absolute or incremental sensitivity, for example, the stimuli vary with respect to intensity, and the required responses are "yes" and "no." In other situations, difference thresholds are measured by presenting stimuli in groups (pairs, triads, etc.) and requiring the subject to respond "same" or "different." To measure temporal resolution, stimuli may be presented in pairs with various interstimulus intervals (ISIs), and the subject responds "one" or "two." Many other applications are possible.

In each of these experimental situations, the probability that the subject will emit one or the other of two response alternatives depends on the value of the stimulus dimension of interest. This relationship is known as the psychometric function. In most cases, the experimenter is not interested in obtaining the entire psychometric function, but only in a point on it corresponding to a criterion response probability $\mathrm{P}$. The stimulus value at this point is known as the "P\% threshold."

Wetherill and Levitt (1965) described several rapid ways to estimate the stimulus value for which the probability of a two-alternative response is at a specified level (the P\% threshold). One algorithm, called Wetherill

This work was supported by NIH Grant EY-02366 to T. Corwin. tracking, proved to combine "extreme simplicity and very high efficiency" (Wetherill \& Levitt, 1965, p. 5). Furthermore, Wetherill tracking "is not critically dependent on the mathematical form of the psychometric function" (Wetherill \& Levitt, 1965, p. 9). We have implemented this algorithm with an Ohio Scientific microcomputer, using a simple BASIC program in conjunction with a short machine-language subroutine. The program yields replicable 50\% thresholds in less than $2 \mathrm{~min}$.

The Wetherill procedure is described as a tracking process because the subject's responses produce changes in stimulus value that converge, toward the end of a session, to the initially selected $\mathrm{P} \%$ threshold. For example, if the selected criterion response probability is $50 \%$, stimuli presented toward the end of a session are close to those for which the subject is equally likely to make either of the two available responses.

Prior to a session, the experimenter specifies the initial stimulus value (i.e., the stimulus level to be presented on the first trial) and the step size (the change in stimulus level to be made on subsequent trials). Although the particular values of these two quantities are not critical, for maximal efficiency the initial stimulus value should approximate the selected response threshold, and the step size should approximate the least noticeable difference between two stimulus levels (Wetherill \& Levitt, 1965, p. 5).

In our implementation, the user may select any of three criterion response probabilities: $29.3 \%, 50 \%$, or $70.7 \%$. In most cases, the $50 \%$ level is most satisfactory, since it yields a threshold value in the smallest number 
of trials. For certain tasks and certain observers, the other response probabilities may yield more reliable thresholds.

Figure 1 is a flowchart of the program. Before each threshold measurement, the experimenter supplies the initial stimulus level $\left(S_{0}\right)$, initial step size $\left(\right.$ delta $\left._{o}\right)$, and the desired criterion response $[\operatorname{Pr}(\mathrm{r})]$. For simplicity, the current program allows only three $P(r)$ values to be selected: $.29, .50$, or .71 . Other values can be obtained,

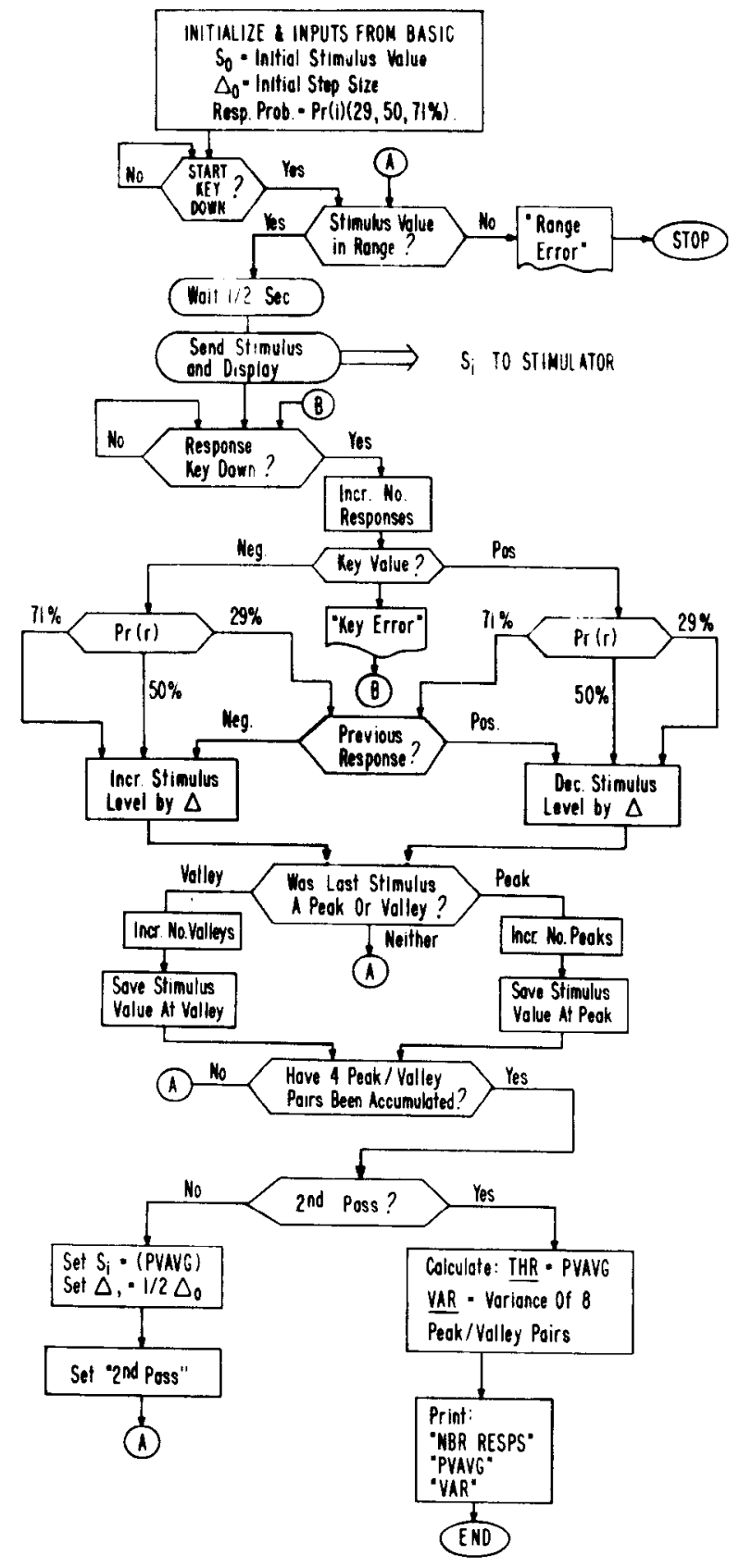

Figure 1. Flowchart of Wetherill tracking program. The program identifies the stimulus level presented on the previous trial (denoted $S_{n-1}$ ) as a local "peak" if it satisfies the relation $\left(S_{n-1}>S_{n-2}\right.$, and $\left.S_{n-1}>S_{n}\right)$. Conversely, a local "valley" has occurred whenever $\left(S_{n-1}<S_{n-2}\right.$, and $\left.S_{n-1}<S_{n}\right)$. but at the cost of increased program complexity. The program requires three binary input lines for responses: These can be built into a three-button switch console held by the subject. One switch (START) is used only for initiating the program. All subsequent stimulus presentations depend on the status of the other two response switches (POS, NEG). The program pauses for $.5 \mathrm{sec}$ after each response before presenting the next stimulus. This pause is required to prevent the observer from setting too fast a pace. After a stimulus has been presented, the program chooses the next stimulus level according to whether the previous response was positive or negative, and according to the criterion response probability being measured. Figure 2 illustrates the contingencies. If $\operatorname{Pr}(r)=.50$, a positive response reduces the next stimulus level by delta $a_{0}$, and a negative response increases the next stimulus level by delta $_{0}$. When $\operatorname{Pr}(r)=.29$ or .71 , the next stimulus level depends on both the current response and the one just preceding it. For $\operatorname{Pr}(r)=.29$, two successive negative responses are needed before the stimulus level is raised, but any positive response lowers it. Conversely, for $\mathrm{P}(\mathrm{r})=.71$, two consecutive positive responses are required to lower the stimulus level, whereas any negative response raises it.

After presenting a stimulus, the program tests to see whe ther the previous stimulus level constituted a change in direction, that is, whether it was a local maximum or minimum. Such points are called "peaks" or "valleys." Only stimulus values at peaks or valleys are used in subsequent threshold and variability calculations.

As recommended by Wetherill and Levitt (1965), the program proceeds until 16 peaks and valleys have occurred. The threshold stimulus level is then calculated as the mean of all peaks and valleys, and the variance of the threshold, based on the variance of the mean values of the eight successive peak-valley pairs, is calculated. The program also records the number of individual responses occurring in the process of a threshold measurement. These three numbers are displayed or printed as the program's final output. In addition, individual stimulus levels are displayed as they are presented, allowing the experimenter to monitor the progress of the program.

The program is executed in two parts, each one accumulating four peak-valley pairs. The first pass begins with the initially specified stimulus level $S_{0}$, and it changes subsequent stimulus levels by the initially specified step size delta ${ }_{0}$. After four peak-valley pairs have been accumulated, a provisional threshold level is obtained by calculating the mean of these eight stimulus levels. The second pass begins by presenting this stimulus level, and all subsequent steps are reduced by $50 \%$ $\left(\right.$ delta $_{1}=.5 \times$ delta $\left._{0}\right)$. The program continues to accumulate four more peak-valley pairs, at which point the final value of threshold, the variance, and the total number of responses are calculated and displayed or printed. 


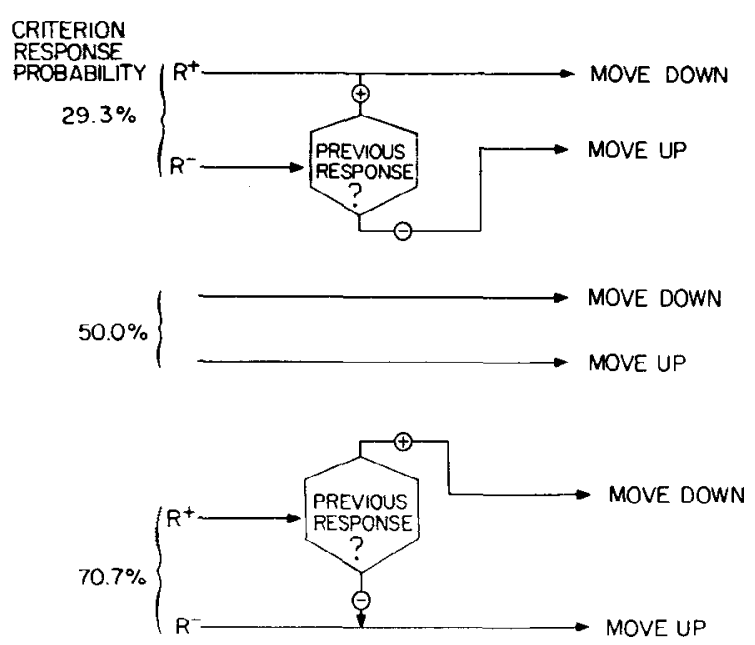

Figure 2. Partial flowchart illustrating the stimulus/response contingencies governing the program's three criterion response probabilities: $\operatorname{Pr}(r)=.29, .50$, or .71 . The subjects' response alternatives are positive (POS) and negative (NEG). When $\operatorname{Pr}(r)=.50$, the current response determines whether the next stimulus level presented is increased (UP) or decreased (DOWN) from its current level. When $\operatorname{Pr}(r)=.29$ or .71 , the next stimulus to be presented depends both on the current response and the one preceding it. By definition, "high" stimulus levels are associated with an increased tendency for the subject to make a positive response, and vice versa.

A criterion $\operatorname{Pr}(r)$ of .50 yields a threshold with a minimum number of trials. Wetherill and Levitt (1965) estimate that an average of two trials is required per peak or valley, so that a threshold determination based on 16 peak-valley pairs would require an average of 32 trials. In confirmation of this estimate, we have obtained 50\% threshold measurements from 25 inexperienced observers and found that an average of $30 \pm 3$ responses was required per threshold. For $\operatorname{Pr}(r)=.29$ or .71 , the average number of trials required per threshold is 48 , an increase of $50 \%$ in subject time.

We have tested the Wetherill tracking program by measuring two-pulse resolution. A stimulus consisted of a pair of 5 -msec pulses of light, and the stimulus variable was the interval between them. The responses, positive and negative, were " 2 " and " 1 ," respectively, depending on how many flashes the observer perceived.

The stimulator was a CRT oscilloscope fitted with a viewing hood. A small circular target spot was produced by disabling the horizontal sweep circuit of the CRT and defocusing the electron beam. Its position was adjusted to a point vertically below the screen's center. The computer output applied a $+5-\mathrm{V}$ step to the y-axis amplifier, the gain of which was adjusted to bring the electron beam precisely to the center of the screen. The screen was then covered with black cardboard containing an opening slightly smaller than the target spot at its center. Thus, the computer output raised the beam from behind the cardboard screen up to the small opening for a precisely calculated duration and then lowered it. Photocell calibrations indicated rise and fall times of less than $.1 \mathrm{msec}$. Stray light internal to the CRT tube proyided a satisfactory fixation source. Observers viewed the screen monocularly through a standard viewing hood (viewing distance $=12$ in.).

Using the Wetherill tracking program, we measured the $50 \%$ thresholds of 28 introductory psychology student volunteers. Each stimulus was a pair of $5-\mathrm{msec}$ pulses separated by a variable ISI. The initial conditions were the same for all subjects: an initial ISI of $50 \mathrm{msec}$ and an initial step size of $6 \mathrm{msec}$. Most subjects had no previous experience with the task, but, for the most part, no difficulty was encountered in understanding or complying with the instructions. Of 28 subjects tested, all but 3 produced thresholds with standard deviations of less than $5 \mathrm{msec}$. The modal value of the withinsubjects standard deviation was $2.3 \mathrm{msec}$ or less. Excluding three subjects with abnormally large variabilities, the average two-pulse resolution for 25 subjects was $58.0 \mathrm{msec}$, with a between-subjects standard deviation of $8.89 \mathrm{msec}$. The average coefficient of variation $(\mathrm{N}=25)$ was $5.06 \%$, and the average number of responses per threshold was approximately $30(\mathrm{SD}=6)$. Most measurements required less than 2 min to complete.

To evaluate the reliability of the tracking method, the thresholds of 11 subjects measured with the Wetherill tracking program were compared with thresholds obtained by a constant stimulus technique. Both measurements were carried out in the same session. The constant stimulus method presented 25 trials at each of eight ISI values in pseudorandom order. A probability. of-seeing curve was plotted from these data, on normal coordinates, fitted with a straight line according to a least-squares criterion, and interpolated to a $50 \%$ level. These threshold values were compared with those obtained with the Wetherill tracking procedure: The correlation between the two procedures was 93 . Constant stimulus thresholds tended to be slightly smaller than Wetherill thresholds: The average difference was 2.9 msec.

Since the Wetherill tracking procedure yields threshold values comparable to those obtained with a constant stimulus procedure but requires only $1 / 6$ as many responses, the Wetherill procedure is clearly efficient. For this reason, and because it is readily understood by inexperienced observers, the procedure should find widespread application in clinical screening tests and in other studies in which it is necessary to test large populations of observers. The procedure is equally applicable to situations in which thresholds must be measured under a variety of experimental conditions within a single session.

A documented program listing is available on request to the first author.

\section{REFERENCE}

Wetherill, G. B., \& LevitT, H. Sequential estimation of points on a psychometric function. British Journal of Mathematical \& Statistical Psychology, 1965, 18, 1-10.

(Received for publication April 19, 1979; revision accepted June 2, 1979.) 